\title{
Topology and generalized shape optimization: Why stress con- straints are so important?
}

\author{
P.Duysinx ${ }^{1 \mathrm{a}}$, L. Van Miegroet ${ }^{1}$, E. Lemaire ${ }^{1}$, O. Brüls ${ }^{1}$, and M. Bruyneel ${ }^{2}$ \\ ${ }^{1}$ LTAS - Department of Aerospace and Mechanics, Institute of Mechanics and Civil Engineering, Université de Liège, 4000 Liege, Belgium \\ ${ }^{2}$ Samtech S.A, Liege Science Park. 4031 Angleur, Belgium
}

Received 23 April 2008, Accepted 13 October 2008

\begin{abstract}
The paper continues along the work initiated by the authors in taking into account stress constraints in topology optimization of continuum structures. Revisiting some of their last developments in this field, the authors point out the importance of considering stress constraints as soon as the preliminary design phase, that is, to include stress constraints in the topology optimization problem in order to get the most appropriate structural layout. Numerical applications that can be solved using these new developments make possible to exhibit interesting results related to the specific nature of strength based structural layout for maximum strength compared to maximum stiffness. This particular character of stress design is clearly demonstrated in two kinds of situations: once several load cases are considered and when unequal stress limits in tension and compression are involved.
\end{abstract}

Key words: Topology optimization, stress constraints, compliant design

\section{Introduction}

Since Bendsøe and Kikuchi [3] have initiated a huge research domain with the so-called topology optimization, literature review (see for instance Bendsøe and Sigmund [4]) shows that the number of works considering stress constraints (see for instance Ref. [8,16, 21, 23]) is rather limited compared to the large number of studies that are based on stiffness problems. More recently a similar observation can be made when considering the recent field of generalized shape optimization using level set methods with pioneer works by Allaire et al. [1], Belytschko et al. [2], Wang and Wang [26]. Following the tracks of topology optimization, this new approach has mainly been focusing on compliance minimization problems than on stress constraints problems. Recently local stress constraints have been included as design restrictions by Van Miegroet and Duysinx [25]. The latter work shows it is possible to solve also the strength problem using a level set description of the geometry and an X-FEM solution for tackling non conforming meshes to the moving boundaries. However this kind of study remains rather seldom.

The 'compliance type' formulation has produced quite interesting results in many problems because controlling the energy and the displacements under the loads is generally good for deflection control and because, for one load case, the compliance minimization leads to a fully stressed design nearly everywhere in the structure (see for instance Rozvany and Birker [18], Rozvany [19, 20]). However there are theoretical results that clearly show that the strongest and the stiffest structural layout can be quite different. For example Rozvany and Birker [18] demonstrated that, for trusses, topology optimization can lead to different results when there are several load cases, different stress limits in tension and compression, or when there are several materials involved.

Therefore, the goal of the paper is to point out the importance of considering stress constraints as soon as the prelimi- nary design phase, that is, to include stress constraints in the topology optimization problem in order to get the most appropriate structural lay-out. Revisiting some contributions of the authors $[8,9,10]$, this paper aims at illustrating the key role of stress constraints in the framework of topology optimization of continuum structures. The developments of Ref. [9] and [10] have extended the work initiated in Ref. [8] to treat:

Integrated stress criteria (i.e. global) relaxed stress constraints that aggregate the stress constraints in each finite element in order to be able to circumvent the large scale character of the local stress constraints [9]. Integrated stress criterions are usual way to consider stress constraints in some work. However this study will show that optimal results obtained do not always have the same character as locally stress constrained designs but, because of the looser control of the stress level get closer to compliance minimization and to stiffness design.

Stress criteria that are able to tackle non equal stress limits in tension and compression [10]. Whereas the von Mises criterion is very usual to predict very precisely failure for ductile materials and metals, von Mises criterion is unable to predict real-life designs when the structure is made of materials with unequal stress limits like concrete or composite materials. One can also remember that thin structural members, like cables or thin sheets, are not able to sustain high compressive loads because of buckling. An indirect procedure to take into account this buckling constraint in the preliminary design phase consists in restricting the compressive loads by reducing the stress limit in compression. From a practical point of view these different behaviors in tension and compression result in quite specific designs.

Numerical applications that can be solved using these new developments make possible to point out interesting results related to the different nature of structural lay out for maximum strength compared to maximum stiffness. This one is

${ }^{\text {a }}$ Corresponding author: $\underline{\text { P.Duysinx } @ \text { ulg.ac.be }}$ 
clearly demonstrated in two kinds of particular situations: once several load cases are considered and when unequal stress limits in tension and compression are involved.

\section{Problem formulation}

\subsection{Maximum stiffness and strength problem formulation}

In the following we consider two kinds of problems. The first one is the classic compliance minimization problem subject to a volume constraint. If the equilibrium equation after discretization by finite element writes $\mathrm{K} \mathrm{qk}=\mathrm{fk}$ where $\mathrm{K}$ is the stiffness matrix, fk and qk are respectively the load vector and the generalized displacement vector of the load case number ' $\mathrm{k}$ ', the minimum compliance problem can be formulated as the following min-max problem:

$$
\begin{array}{cc}
\min _{0 \leq \rho(x) \leq 1} & \max _{k} f_{k}^{T} q_{k} \\
\text { s.t. } & V=\int_{\Omega} \rho(x) \square \quad d x \leq \bar{V}
\end{array}
$$

$\rho(x)$ is the material density at point $x$. Compliance minimization problem gives rise to the stiffest structural design for the given load case measured in energy norm.

The second problem considered is the problem of maximum strength. In the following the maximum strength problem is the stress constrained optimization problem. In this study strength problem will be formulated as the following: finding the structural topology design that supports the applied loads using a minimum amount of material while avoiding the material failure everywhere in the structure.

$$
\begin{array}{cc}
\min _{0 \leq \rho(x) \leq 1} & \int_{\Omega} \rho(x) d x \\
\text { s.t. } & \left\|\sigma^{e q}(\rho(x), x)\right\| \leq T \quad \text { if } \rho(x)>0
\end{array}
$$

where $\left\|\sigma^{e q}(\rho(x), x)\right\|$ is an equivalent stress criterion which predicts the failure of the material at point $x$ while $T$ is the stress limit. Usually Von Mises criterion is considered, but we can also use other criteria in order to take into account different stress limits in tension and compression. Please note also that, in this study, a pure elastic regime is assumed, so that the criterion predicts the end of the elastic behavior everywhere in the material (and in the microstructure).

\subsection{Topology optimization problem}

Generalized shape optimization of structures without any a priori on the structural topology can be achieved by formulating the problem as an optimal material distribution on a given design domain (see Bendsøe and Sigmund [4]). As the optimal material distribution problem is generally solved numerically using a finite element discretization approach, the design domain is divided into finite elements and a density variable is attached to each element. The optimal material distribution problem could be solved as a discrete valued problem, but this approach is very complicated because of its highly combinatorial nature. Here this highly combinatorial formulation is avoided by considering an alternative formulation in which we allow the density parameter running continuously from void to solid via all intermediate densities, so that composite materials of intermediate densities are included in the design. The continuous formulation presents the advantage to allow using sensitivity analysis and mathematical programming algorithms to solve the problem in an efficient way. Unfortunately the discretized problem is ill-posed and its numerical solutions are mesh-dependent. To overcome the difficulty, we use here a restriction method of the design space based on a bound over the perimeter [13], a low-pass filtering scheme [22], or a combination of both.

The modelling of the intermediate density properties is based on the power-law model (also called SIMP model [4]). If the script $*$ denotes the effective properties of the porous material and the index 0 is relative to the solid material properties, the effective Young's modulus $E^{*}$ is given in term of the density $\rho$ by:

$$
E^{*}=\rho^{p} E^{0}
$$

The factor $p$ is introduced to penalize the intermediate densities in order to end up with 'black and white' designs. Moreover to consider stress constraints in continuous topology optimization, one also needs the definition of a relevant stress measure in the porous composites. Following the approach developed in Duysinx and Bendsøe [8] we consider an overall stress measure that controls the stress state in the microstructure. For the SIMP model of stiffness, a careful study [8] showed that a power-law model with the same power $p$ is a consistent model for the micro-stresses $\sigma_{i j}$ :

$$
\sigma_{i j}=\sigma_{i j}^{*} / \rho^{p}
$$

Therefore if the failure is predicted by a quadratic failure criterion $\left\|\sigma^{e q}\right\|$ like von Mises equivalent criterion, the first failure in the microstructure of the porous composites is predicted by the following criterion in terms of effective stresses:

$$
\left\|\sigma^{e q}(\rho)\right\|={ }^{*} \sigma^{e q} / \rho^{p} \leq T
$$

\section{Stress constraints in topology optimiza- tion}

\subsection{Stress singularity phenomenon}

At first it is interesting to remind the reader with a very important difficulty that arises when dealing with stress constraints in topology optimization. As it has been shown for instance in Kirsch [14] and in Cheng and Jiang [6], topology optimization with stress constraints is subject to the so-called 'singularity phenomenon'. At short the paradox comes from the fact that the optimization procedure is often unable to remove or to add some vanishing members without violating the stress constraints although one would end up with a perfectly feasible design if they were removed or added. From a mathematical point of view, the classical gradient based math programming algorithms, which rely on the Karush-KuhnTucker optimality conditions, are unable to reach some optimum configurations because of the degeneracy of the design space. In order to turn around the difficulty, one has to use a perturbation technique of the stress constraints, generally known as the relaxation technique (Cheng and Guo [7]) that results in a relaxation of the stress limits in the low-density regions. In this paper we use the following relaxed formulation of the overall stress criterion (see Ref. [8]): 


$$
\frac{1}{T} \frac{\sigma^{e q}}{\rho^{p}}-\frac{\varepsilon}{\rho}+\varepsilon \leq 1
$$

This relaxed formulation of the stress constraints is considered in the numerical solution of the topology optimization problem with stress constraints. The solution procedure requires solving of a sequence of perturbed problems with decreasing values of the parameter $\varepsilon$.

\subsection{Integrated global stress criterion}

In Duysinx and Bendsøe [8] the stress is treated as a local constraint, i.e. for every finite element. This formulation offers a full control of the stress state, but it also dramatically increases the size of the optimization problem and thus the computation time of the solution. Here we propose an alternative approach based on an integrated version of the relaxed failure criterion. The global stress constraint (i.e. integrated constraint) simplifies the computational complexity of the optimization problem at the expense of a weaker control of the local stress state. A key issue is to take into account the 'singularity phenomenon' of the stress constraints. So an original aspect is to include simultaneously the

-relaxation technique that alleviates the singularity phenomenon and the use of effective stress criteria into the global stress constraint.

We propose to consider two global measures of the relaxed distributed stress criterion. The first global constraint is the ' $q$-norm' of the relaxed stress criterion (6); the second one is the ' $q$-mean' of the relaxed stress criterion (7).

$$
\begin{gathered}
{\left[\sum_{e=1}^{N}\left(\max \left\{0, \frac{1}{T} \frac{\left({ }^{*} \sigma^{e q}\right)_{e}}{\rho_{e}^{p}}-\frac{\varepsilon}{\rho_{e}}+\varepsilon\right\}\right)^{q}\right]^{1 / q} \leq 1} \\
{\left[\frac{1}{N} \sum_{e=1}^{N}\left(\max \left\{0, \frac{1}{T} \frac{\left({ }^{*} \sigma^{e q}\right)_{e}}{\rho_{e}^{p}}-\frac{\varepsilon}{\rho_{e}}+\varepsilon\right\}\right)^{q}\right]^{1 / q} \leq}
\end{gathered}
$$

To prevent negative valued relaxed criteria from contributing to the norms, one must consider the maximum value of the relaxed stress criterion and zero. Negative values of the relaxed criterion appear only for low stressed elements and thus can be truncated without influencing the global constraint. Furthermore, the function remains continuous up to derivative ' $q-1$ ', so that the constraint remains sufficiently smooth. A major advantage of ' $q$-norm' and ' $q$-mean' functions comes from the fact that they make possible to set bounds of the maximum value of the relaxed criterion by upper or by lower values. The ' $q$-norm' is an upper bound of the maximum value of the criterion while the ' $q$-mean' is a lower bound of the maximum local value of the criterion. In addition this gap closes to 0 when ' $q$ ' value is going to infinity. So sharp bounding needs to take high values of ' $q$ ' parameter. However in practice, we take $q=4$ because it allows controlling weakly the maximum value of the stress criterion while avoiding ill-conditioned optimization problems.

\subsection{Different stress limits in tension and com- pression}

As in most of metallic structural problems, stress constrained topology optimization is usually based on the qua- dratic von Mises criterion, which predicts the end of elastic behavior. However Von Mises is inappropriate to predict different behaviors in tension and compression. For this kind of behavior, one requires particular failure criteria that are able to cope with unequal stress limits as in studies related to composite materials or concrete and rocks. Based on the work of Laschet [15] and Boresi et al. [5] that reviewed different criteria that are able to cater with unequal compressive and tensile stress limits, we have selected two criteria generally used in prediction of plastic design of adhesive materials: Rhagava [17] and Ishai [12] criteria. Both are quadratic criteria which predict a different behavior in tension and compression through the dependency of the criterion upon the first invariant of the stresses. Let's remind the reader with the definition of the first stress invariant $J_{1}=\sigma_{i i}$ and the second deviator stress invariant $J_{2 D}=0.5 s_{i j} s_{i j}$. The second deviator stress tensor is directly related to the distortional shear stress $\tau_{0}$ and to the equivalent von Mises stress $\sigma_{V M}: 3 J_{2 D}=\tau_{0}^{2}=\sigma_{V M}^{2}$. The presence of the second invariant is obvious because the two criteria have to recover the von Mises criterion when the stress limits are equal. The first invariant is related to the hydrostatic pressure $J_{1}=3 \sigma_{h}$ and its presence in the criterion is essential since it introduces the dependence upon the sign of the stress state and hence the different behaviors in tension and compression.

The Rhagava [17] and Ishai [12] stress criteria and the related equivalent stress can be written as:

$$
\begin{aligned}
& \sigma_{R A G}^{e q}=\frac{J_{1}(s-1)+\sqrt{J_{1}^{2}(s-1)^{2}+12 J_{2 D} s}}{2 s} \leq T \\
& \sigma_{I S H}^{e q}=\frac{(s+1) \sqrt{3 J_{2 D}}+(s-1) J_{1} \leq T}{2 s} \leq T
\end{aligned}
$$

In which we have used the following notations: $T$ and $C$ are the stress limits (in absolute value) respectively in tension and compression, while ' $s$ ' is the ratio between the stress limits in compression and in tension: $s=C / T$.

As it is demonstrated in Laschet [15], the Raghava criterion can also be viewed as a particular case of Tsai-Wu criterion of orthotropic materials when one assumes that the stress limits along the 3 orthotropy directions are the same. The Ishai criterion belongs to the Drucker-Prager plasticity criterion family that is of a general use for chalk, rocs and soils materials. The two criteria can be compared in Fig. 1 and 2 where their failure envelopes are plotted in the space $\left(\sigma_{\mathrm{h}}, \tau_{0}\right)$ of the hydrostatic pressure and of the distortional shear stress. At first the failure surface of the Raghava approach is a parabolic curve whereas the Ishai criterion is a hyperbolic one. But from the figures, one also notices that the two theories predict the failure for different maximum hydrostatic pressures and for different pure distortional shear stresses. The pure hydrostatic stress limit predicted by the Ishai theory is twice the Raghava's one. We remind the reader that this hydrostatic failure stress is infinite in von Mises theory. Failure under a pure shear stress state is also different in the two theories. The pure distortional shear stress limit for Raghava's theory is always greater than Ishai's one because $\sqrt{s} \geq 2 s /(s+1)$ 


\section{Implementation and sensitivity analysis}

The implementation of topology optimization including stress constraints has been realized in computer code coupling a finite element analysis code, its sensitivity analysis and an optimization solver based on the sequential convex programming approach. The finite element code has 4-node and 9-node plane stress finite elements. The optimization is based here on the CONLIN solver by Fleury [13]. This architecture is now classical. However because of the large number of stress constraints to consider, there is a general interest in describing the guidelines of the implementation of the sensitivity analysis of stress criteria. Here the work is detailed for the Ishai and Raghava criteria that generalize von Mises criterion $(s=1)$.

The equilibrium equation after discretization by finite element writes $\boldsymbol{K} \boldsymbol{q}=\boldsymbol{f}$ where $\boldsymbol{K}$ is the stiffness matrix, $\boldsymbol{q}$ is the generalized displacement vector and $\boldsymbol{f}$ is the load vector. In a vector form the macroscopic stress in the finite element is given by $\boldsymbol{\sigma}=\boldsymbol{T} \boldsymbol{q}$ where $\boldsymbol{T}$ is the stress matrix of the element. The two first invariants of the stress in the finite element that are required to calculate the equivalent stress criteria can also be written in matrix form:

$$
\begin{aligned}
& J_{1}^{*}=3 \sigma_{h}^{*}=w^{T} \sigma=W q \\
& J_{2 D}^{*}=1 / 3\left(\sigma_{V M}^{*}\right)^{2}=1 / 3 q^{T} V q
\end{aligned}
$$

As the stress matrix depends linearly upon the stiffness properties, one can also write $T=\rho^{p} T^{0}$ where $\boldsymbol{T}^{0}$ is the stress matrix of an element of relative density of 1 . In the same way the influence of density can be put in evidence in matrices $\boldsymbol{W}$ and $\boldsymbol{V}: V=\rho^{2 p} V^{0}$ and $W=\rho^{p} W^{0}$ where matrices

$$
\frac{\partial\left\|\sigma_{R A G}^{e q}\right\|}{\partial \rho_{i}}=\left\{\frac{s-1}{2 s} \frac{\left\|\sigma_{R A G}^{e q}\right\|}{\sqrt{(s-1)^{2}\left(W^{0} q\right)^{2}+4 s q^{T} V^{0} q}} W^{0}\right.
$$

In a direct approach of the sensitivity analysis one would evaluate all the derivatives of the generalized displacements $\partial q / \partial \rho_{i}$, which could be a major work in topology optimization. However the number of active stress constraints is generally smaller than the number of design variables and the adjoined method is generally preferred because only one additional load case is required per active constraint. In this case the adjoined load vector is the vector that is put between curly braces.

One will also notice that a strategy to delete stress constraints that are likely not to be active during the optimization iteration is very useful to reduce as much as possible to dimension of the optimization problem. Stress constraints are simply evaluated and we save the sensitivity analysis.

\section{Numerical applications}

\subsection{Two-bar truss problem}

The first application is the classic two-bar truss problem. Despite its simplicity the two-bar truss problem is an interesting example to illustrate the difficulties of stress constraints in topology optimization. Indeed even if the lay-out of the solution is the obvious two-bar truss for equal stress constraints and the von Mises stress criterion (see Fig. 3 top), the $V^{0}$ and $W^{0}$ are respectively the von Mises stress matrix and the hydrostatic stress matrix of the solid element.

Therefore one can evaluate easily the effective failure criteria based on Ishai and Raghava theories with the following expressions:

$$
\begin{gathered}
\left\|\sigma_{I S H}^{e q}\right\|={ }^{*} \sigma_{I S H}^{e q} / \rho^{p} \\
=\frac{s-1}{2 s} W^{0} q+\frac{s+1}{2 s} \sqrt{q^{T} V^{0} q} \\
\left\|\sigma_{R A G}^{e q}\right\|={ }^{*} \sigma_{R A G}^{e q} / \rho^{p} \\
=\frac{(s-1) W^{0} q+\sqrt{(s-1)^{2}\left(W^{0} q\right)^{2}+4 s q^{T} V^{0} q}}{2 s}
\end{gathered}
$$

Sensitivity analysis can also be implemented efficiently for Ishai and Raghava criteria as it can be done for Von Mises theory. As only the guidelines of the implementation are presented we give only the main results of the derivation calculus.

For Ishai equivalent stress, we get:

$$
\begin{aligned}
& \frac{\partial\left\|\sigma_{I S H}^{e q}\right\|}{\partial \rho_{i}} \\
& =\left\{\frac{s-1}{2 s} W^{0}+\frac{s+1}{2 s} \frac{1}{\sqrt{q^{T} V^{0} q}} V^{0} q\right\}^{T} \frac{\partial q}{\partial \rho_{i}}
\end{aligned}
$$

Raghava criterion is a bit more difficult. After some calculus the derivative of the averaged stress criterion can be written as:

$\left.W^{0}+\frac{2}{\sqrt{(s-1)^{2}\left(W^{0} q\right)^{2}+4 s q^{T} V^{0} q}} V^{0} q\right\}^{T} \frac{\partial q}{\partial \rho_{i}}$

one have to be able to overcome the problem of the singular stress paradox. The part of the design domain that is enclosed inside the two bars remains highly strained and thus highly stressed while all its material vanishes. Without the relaxation technique it is impossible to remove totally this part.

In the framework of unequal stress limits the second point of interest is to be able to check the analytical solution for this problem. The analytical solution has been exhibited by Rozvany [19] while discussing shortcoming of Michell's truss theory. When adopting a tensile stress limit is 3 times higher than the compressive stress limit, the solution is still a two bar truss, but the two bars make an angle of $30^{\circ}$ and $60^{\circ}$ degrees respectively with the foundation wall. Ishai equivalent stress is adopted to take into account the different stress limits effect. The optimized material distribution is presented in Fig.3 (lbottom). The numerical result totally matches the theoretical prediction, which validates the method. It also shows immediately that stress constraints can modify at least the optimal solution of topology optimization, at least in terms of shape.

\subsection{Three-bar truss problem}

The 3-bar truss problem is very well suited to illustrate the specific character of maximum strength versus stiffness de- 
sign when considering several load cases in topology optimization. The geometry of the problem is given in Figure 4. The sizes and material data of the benchmark are normalized: $\mathrm{L}=1 \mathrm{~m}, \mathrm{~W}=2.5 \mathrm{~m}, \mathrm{E}=100 \mathrm{~N} / \mathrm{m}^{2}, \mathrm{v}=0.3$. Three load cases (with different magnitudes and orientations) are applied at the center of the free edge. The design domain is meshed with $50 \times 20$ finite elements. This means that for local stress constraints one has to deal with $3 \times 1000$ restrictions. The volume bound is set to $25 \%$ of the design domain area.

At first, the minimum compliance design (minimum of the maximum of the compliance for the three load cases) is studied (Figure 5a). The optimal topology is clearly a 2-bar truss. Compliances for the 3 load cases are equal to $73.3 \mathrm{Nm}$. A posteriori computation of the stress level in the two-bar truss solution shows that the stress level is quite high. The maximum value of the local von Mises criterion varies from $228 \mathrm{~N} / \mathrm{m}^{2}$ to $571 \mathrm{~N} / \mathrm{m}^{2}$ per load case. In a second optimization run one investigates the strength problem and one considers local von Mises stress constraints with a stress limit of $150 \mathrm{~N} / \mathrm{m}^{2}$ and one obtains the topology of Figure 5b, which is a 3-bar truss. The compliances of the minimum stress design solution are a bit bigger than minimum compliance solution $(91.2 \mathrm{Nm}, 45.6 \mathrm{Nm}$ and $45.0 \mathrm{Nm}$ for load cases 1,2 and 3). This means that the compliance design (a two-bar truss) with nearly the same volume is overstressed by nearly a factor 1.5 compared to the maximum strength design. This also shows that the three-bar truss is not a local optimum but clearly an alternative design to the two -bar truss with a different topology. This also points out that compliance designs and minimum stress designs can lead to different topology solutions when there are several load cases.

One can further compare the local stress constraint formulation and the alternative approaches based on global restrictions ('q-mean' and ' $q$-norm' with q=4) with optimization runs presented in Figures 6. One has the solution when minimizing the volume of material with a (heuristic) bound of $92 \mathrm{~N} / \mathrm{m}^{2}$ over the ' $q$-mean' of relaxed von Mises stresses (Figure 6a). The area of the optimal design solution is 0.56 $\mathrm{m}^{2}$. In Figure $6 \mathrm{~b}$, the bound over ' $q$-norm' is set to $500 \mathrm{~N} / \mathrm{m}^{2}$ and the optimal area is $0.62 \mathrm{~m}^{2}$. Topology results, which are 2-bar truss designs in both cases, look more like the compliance design and not like the local stress constrained design. This suggests that once we lose the control over the local stress state, we get closer to a global criterion like the minimum compliance design. However considering the stress constraints even in a "globalized way" is favorable to reduce the maximum local stress values compared to the compliance design. For ' $q$-mean' one has the following maximum von Mises stresses: $237 \mathrm{~N} / \mathrm{m}^{2}, 215 \mathrm{~N} / \mathrm{m}^{2}$, and 207 $\mathrm{N} / \mathrm{m}^{2}$ for the first, second and third load cases. For the ' $q$ norm' on has a maximum von Mises stresses of $230 \mathrm{~N} / \mathrm{m}^{2}$, $235 \mathrm{~N} / \mathrm{m}^{2}$, and $231 \mathrm{~N} / \mathrm{m}^{2}$ respectively for the three load cases. One advantage of the stress constraint global approach is to allow saving one or two orders of magnitude in the optimizer solution time compared to the local stress approach, but we lose the very specific character of the stress constrained design.

Finally one can exploit the example to appreciate the effect of unequal stress limits in tension and compression and Ishai criterion. Figure 7 shows the optimum material distribution for unequal stress limits with Ishai criterion $(\mathrm{T}=150$ $\mathrm{N} / \mathrm{m}^{2}$ and $\mathrm{C}=450 \mathrm{~N} / \mathrm{m}^{2}$ in Fig 7left and $\mathrm{T}=450 \mathrm{~N} / \mathrm{m}^{2}$ and $\mathrm{C}=150 \mathrm{~N} / \mathrm{m}^{2}$ in Fig.7 right). Both optimal lay-out configura- tions are once again two-bar trusses but their geometry is different in order to make use of the better strength respectively in tension or in compression. When the material is very resistant to compressive stresses, middle and left bars merge. Conversely when material is very good in tension, the optimal structure looks like a cable structure. This shows that real characteristics of the problem, here the unequal properties in tension and compression, may strongly influence topology results.

\subsection{Four-bar truss problem}

The third example stems from an application initially suggested by Swan and Kosaka [24] to demonstrate that ultimate strength optimization can lead to substantially different results from a minimum elastic compliance design. In the present study the same example is revisited in the framework of first failure stress constraints and elastic behavior. The design domain is a square $(\mathrm{L}=1 \mathrm{~m})$ clamped in its four corners. A unit load is applied downwards in the center of the square domain. Using a normalized material $\left(E=100 \mathrm{~N} / \mathrm{m}^{2}\right.$ and $v=0.3$ ) one bounds at first the von Mises equivalent stress to $\mathrm{T}=\mathrm{C}=10 \mathrm{~N} / \mathrm{m}^{2}$ (see Fig 8-left). The same optimal topologies could be obtained for minimum compliance as for minimum volume subject to stress constraints. It is cross-like structure that withstands the load in tension (upper members) and compression (lower members). Now if one uses a material that has a better strength limit in compression than in tension ( $\left.\mathrm{T}=6 \mathrm{~N} / \mathrm{m}^{2}, \mathrm{C}=24 \mathrm{~N} / \mathrm{m}^{2}\right)$, the optimal structure (Fig 8-center) is an arch that works only in compression. Conversely if the material works better in tension than in compression $\left(\mathrm{T}=24 \mathrm{~N} / \mathrm{m}^{2}, \mathrm{C}=6 \mathrm{~N} / \mathrm{m}^{2}\right)$ one gets a structure (Fig. 8-right) working exclusively in tension like cables in suspension bridges. We have also to remark that the results for unequal stress limits have been made with the Ishai criterion, but the same results can be obtained with the Raghava criterion since they give exactly the same strength for members under a one dimensional stress state as here.

\section{Conclusion and perspectives}

Based on some developments of topology optimization of continuum structures including stress constraints, this work has provided several illustrative examples of the conjuncture that structural lay-out may be different for maximum stiffness and strength as soon as we have several load cases (even in metallic materials) and / or when we have a different behavior in tension and compression. The conclusion of the work leads to stress that designers have to pay attention to the relevant constraint for their problems as soon as the preliminary design phase and to include them early in the design process.

The specific character of structural lay-outs when including stress constraints encourages the authors to develop further research work in order to cope efficiently with local constraints in many design problems such MEMS design, material microstructure tailoring, etc. Stress constraints states also several challenges for research such developing solution algorithms able to handle more efficiently optimization problems with fast growing size rising up to one million of design variables and several hundred thousand of constraints. 


\section{Acknowledgments}

Part of this research has been realized under research project ARC 03/08-298 'Modeling, Multiphysics Simulation and Optimization of Coupled Problems - Application to Micro ElectroMechanical Systems' supported by the Communauté Française de Belgique. The authors want to thank Professor C. Fleury for making the CONLIN software available.

\section{References}

1. G. Allaire F. Jouve, A.M. Toader, Structural optimization using sensitivity analysis and a level-set method. Journal of Computational Physics. 194, 363-393 (2004).

2. T. Belytschko, S. Xiao, C. Parimi, Topology optimization with implicit functions and regularization. Int. J. Num. Meth. in Engng, 57, 1177-1196 (2003).

3. M.P. Bendsøe, N. Kikuchi, Generating Optimal Topologies in Structural Design Using a Homogenization Method. Computer Methods in Applied Mechanics and Engineering, 71, 197-224 (1988).

4. M.P. Bendsøe, O. Sigmund, Topology optimization: theory, methods, and applications. (Springer Verlag 2003).

5. A.P. Boresi, R.T. Schmidt, O.M. Sidebottom, Advanced Mechanics of Materials. (5th Edition. John Wiley and Sons, New York 1993).

6. G.D. Cheng, Z. Jiang, Difficulties in truss topology optimization with stress constraints. Eng. Optim., 20, 129148 (1992).

7. G.D. Cheng, X. Guo, e-relaxed approach in structural topology optimization. Struct. Opt., 13, 258-266 (1997).

8. Duysinx P. and M.P. Bendsøe. (1998). Topology optimization of continuum structures with local stress constraints. In: Int. J. Num. Meth. Engng., 43, 1453-1478.

9. P. Duysinx, O. Sigmund. New developments in handling stress constraints in optimal material distribution. In: proceedings of 7th Symposium on Multidisciplinary Analysis and Optimization A1AA-98-4906, 1501-1509 (1998).

10. P. Duysinx, Topology optimization with different stress limit in tension and compression, In: Proceedings of the Third World Congress of Structural and Multidisciplinary Optimization WCSMO3, Buffalo (NY-USA 1999).

11. C. Fleury, Mathematical programming methods for constrained optimization: dual methods. In: volume 150 of Progresses in Astronautics and Aeronautics (M.P. Kamat ed.) 183-208, AIAA (1993).

12. S. Geli, G. Dolex, O. Ishai, An effective stress/strain concept in the mechanical characterization of the structural adhesive bonding. Int. J. of Adhesion \& Adhesives, 1981, 135-140 (1981).

13. R.B. Haber, C.S. Jog, M.P. Bendsøe, A new approach to variable-topology shape design using a constraint on perimeter. Struct. Opt., 11, 1-12 (1996).

14. U. Kirsch, On singular topologies in optimum structural design. Struct. Opt. 2, 133-142 (1990).

15. G. Laschet, Prédiction par eléments finis du comportement non linéaire et de la résistance d'assemblage multimatériaux collés. Ph.D. thesis. University of Liège (in French, 1991).

16. J.T. Pereira, E.A. Fancello, C.S. Barcellos, Topology optimization of continuum structures with material failure constraints. Structural Multidisciplinary Optimization 26, 50-66 (2004).

17. R. Raghava, R.M. Caddel, G.S. Yeh, the macroscopic yield behaviour of polymers. Journal of Material Sciences, 8, 225-232 (1973).

18. G.I.N. Rozvany, T. Birker, On singular topologies in exact layout optimization. Struct. Opt., 8, 228-235 (1994).

19. G.I.N. Rozvany, Some shortcomings in Michell's truss theory. Struct. Opt., 12, 244-250 (1996).

20. G.I.N Rozvany, Exact analytical solutions for some popular benchmark problems in topology optimization. Struct. Opt., 15, 42-48 (1998).

21. P.Y. Shim, S. Manoocheri. Generating optimal configurations in structural design using simulated annealing. International Journal for Numerical Methods in Engineering. 40, 1053-1069 (1997).

22. O. Sigmund. On the design of compliant mechanisms using topology optimization, Mechanics of Machines and Structures, 25, 493-526 (1997).

23. M. Stolpe, K. Svanberg. On the trajectory of the erelaxation approach for stress-constrained truss topology optimization. Structural Multidisciplinary Optimization 21, 140-151 (2001).

24. C.C. Swan, I. Kosaka, Voigt-reuss topology optimization for structures with nonlinear material behaviors. International Journal for Numerical Methods in Engineering, 40, 3785-3814 (1997).

25. L. Van Miegroet P. Duysinx. Stress concentration minimization of $2 D$ filets using $X$-FEM and level set description. Structural Multidisciplinary Optimization, 33, 425438 (2007).

26. M. Wang, X. Wang, D. Guo. A Level Set method structural topology optimization. Comp. Methods in Appl. Mech Engng, 191:227-246 (2003). 